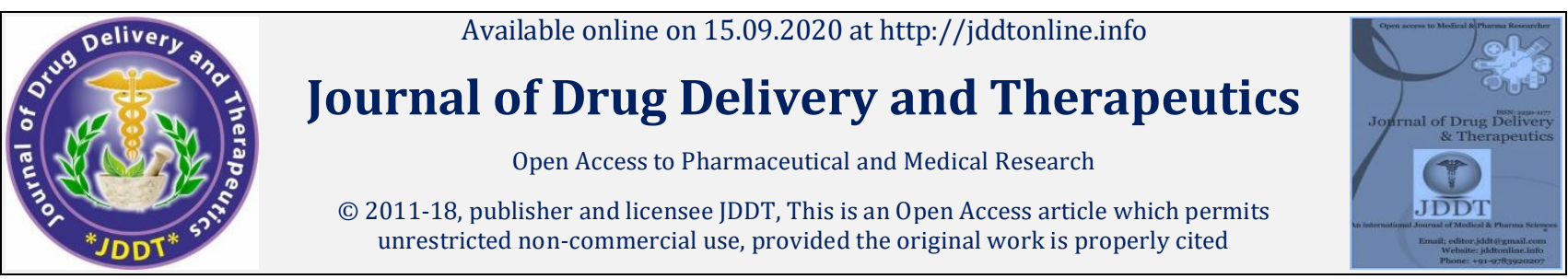

Open $\odot$ Access

Review Article

\title{
A Review on Pharmacological Activities of Lupeol and its Triterpene Derivatives
}

\author{
Nitika Sharma, Priyankul Palia*, Amit Chaudhary, Shalini, Kritika Verma, Inder Kumar \\ School of Pharmacy, Abhilashi University, Mandi, HP, India
}

\begin{abstract}
Lupeol is a pentacyclic triterpenoid commonly distributed in the plant kingdom and is found in edible fruits and vegetables. It is a naturally occurring triterpene that is used to reduce the inflammatory responses and also have immunomodulating properties. Lupeol and its derivatives have a great potential to act as an anti-inflammatory, anti-microbial, anti-proliferative, anti-invasive, anti-angiogenic, antiprotozoal, and cholesterol-lowering agent. Various studies have shown that anti-inflammatory activity of lupeol through the modulation of p38 pathways inhibits neuroinflammation in the cerebellum and induces neuroprotection. It has been also found effective on lung cancer (i.e A427 cancer cells and normal MRC-5 cells). Observation of inhibiting the growth of lung cancer cells is checked by MTT assay. Lupeol and its ester lupeol linoleate have been used to reduce the levels of hypercholesterolemia in the rats and decrease the activities of such enzymes namely $\mathrm{Na}+, \mathrm{K}+-\mathrm{ATPase}, \mathrm{Ca} 2+-\mathrm{ATPase}$, and $\mathrm{Mg} 2+$. Lupeol also decreases the levels of calcium-oxalate and has cytoprotective action against free-radical-induced damage and also decreases the level of cadmium in the kidney.
\end{abstract}

Keywords: Lupeol, anti- inflammatory, anti-protozoal, triterpenoids

Article Info: Received 12 July 2020; Review Completed 17 August 2020; Accepted 23 August 2020; Available online 15 Sep 2020

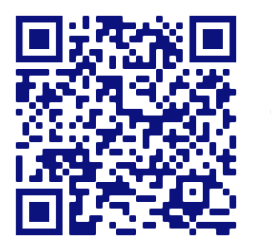

\section{Cite this article as:}

Sharma N, Palia P, Chaudhary A, Shalini, Verma K, Kumar I, A Review on Pharmacological Activities of Lupeol and its Triterpene Derivatives, Journal of Drug Delivery and Therapeutics. 2020; 10(5):325-332

http://dx.doi.org/10.22270/jddt.v10i5.4280

Priyankul Palia, School of Pharmacy, Abhilashi University, Mandi, HP, India

\section{INTRODUCTION}

There is day by day growing interested in natural triterpenoids, also known as phytosterols. From ancient times natural products are used as remedies to treat human diseases. Triterpenoids are called secondary metabolites and their pharmacological activities are derived from plants, fruits fungi, etc. ${ }^{1}$ Pharmacological importance, of medicinal plants are the god gift of a healthy lifestyle. More than 8000 different plant species are used in a different part of the world for the treatment of many disorders. Biosynthetically rearrangements of squalene epoxide various triterpenoids are synthesized. Various processes like oxygenation, hydrogenation, and dehydrogenation synthetically obtain hydrocarbon triterpenoid derivatives. Among triterpenoids have a double bond at position 20. It is found in different species of plants including Emblica Officinalis, Bombax ceiba, Walsura trifolita, and etc. ${ }^{2}$

Emblica Officinalis (Family: Phyllanthaceae) commonly known as Amla or Indian gooseberry. In Ayurveda, it is the most important medicinal plant for the prevention of various ailments. It is widely distributed in tropical and subtropical countries like China, India, Indonesia, and Southeast Asia. Its fruits contain many chemical constituents and a higher amount of polyphenols like gallic acid, ellagic acid, different tannins, minerals, vitamins, amino acids, fixed oils, and flavonoids like quercetin. E. officinalis having a rich amount of vitamin C (478.56 mg/100 ml) as compared to other fruits, e.g. apple, lime, pomegranate. 


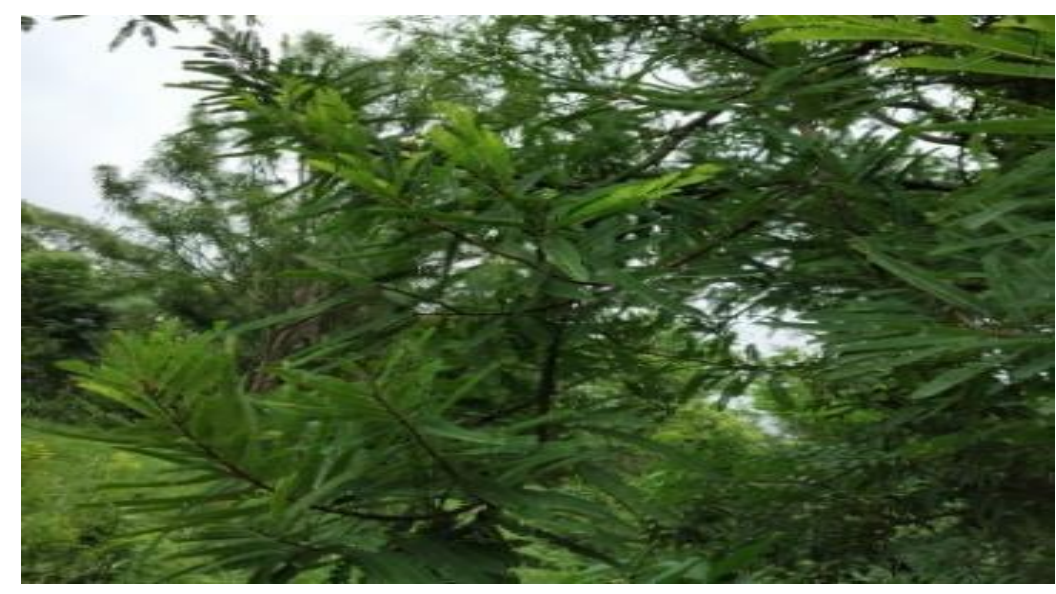

Figure 1: Diagram of Amla Tree

\section{LUPEOL}

Lupeol is the pentacyclic triterpenoids. ${ }^{4}$ Many derivatives are synthesized from the lupeol. It is broadly distributed in the plant kingdom and is found in edible fruits and vegetables. It has great potential to act as an antiinflammatory, anti-microbial, anti-protozoal, antiproliferative, anti-invasive, anti-angiogenic, and cholesterol-lowering agent. According to studies of in vitro and in vivo models, it has also been tested for its biological activities against conditions including wound healing, diabetes, cardiovascular disease, kidney disease, andarthritis. 5

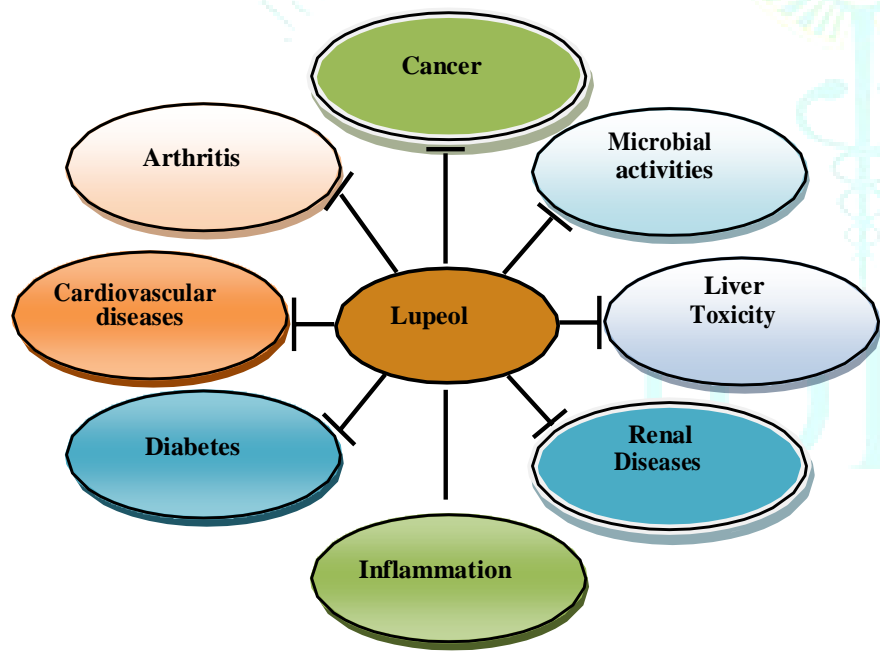

Figure 2: Diagram represents the effects of lupeol for different type of diseases

\section{CHEMICAL STRUCTURE AND ANALYSIS}

The structure of Lupeol is represented in Fig.3. The chemical formula of lupeol is $\mathrm{C} 30 \mathrm{H} 50 \mathrm{O}$ and its melting point is $215-216^{\circ} \mathrm{C}$ and the molecular weight of lupeol is 426.7174 ( $\mathrm{g} / \mathrm{mol}$.). Lupeol in their infra-red spectrum shows the presence of a hydroxyl function and an olefinic moiety at 3235 and $1640 \mathrm{~cm}^{-1}$, respectively. The presence of seven methyl singlet and an olefinic function in the ${ }^{1} \mathrm{H}$ NMR spectrum may be triterpenes. By using (HPLC) method and mass spectrometric (MS) showed that lupeol exhibits a parent ion peak at $m / z 409[\mathrm{M}+\mathrm{H}-18]^{[+]}{ }^{6}$

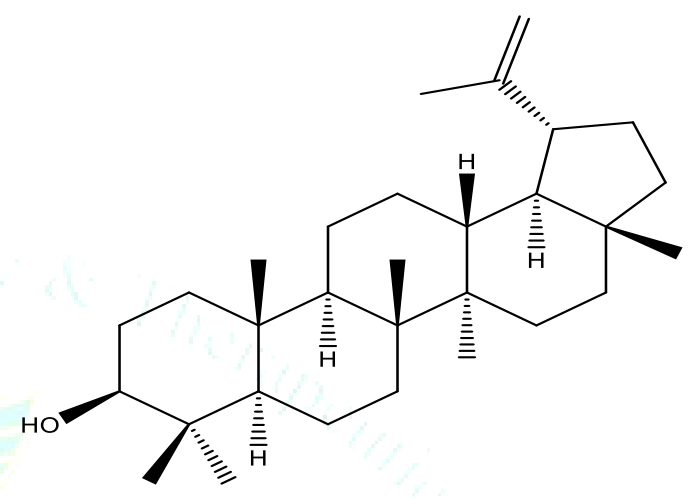

Figure 3: Chemical structure of Lupeol

\section{Lupeol acts as an anti-inflammatory}

Today, inflammatory diseases have become a major problem in the whole world and cause health issues that are dangerous for the human body. There is the development of natural products and their synthetic analogs which are useful for acute and chronic anti-inflammatory activity. Usually, there are only four types of signs and symptoms redness, sneezing, swelling, and pain against any pathogen or injury. The most important factor is Nuclear factor-kappa B (NF- $\mathrm{kB}$ ) which is responsible for the inflammatory responses. Lupeol is a naturally occurring triterpene which is used to reduce the inflammatory response and have immunomodulating properties. ${ }^{7}$ The various studies have been showing that anti-inflammatory activity of lupeol through the modulation of p-38 pathways inhibits neuroinflammation in the cerebellar and induces neuroprotection. ${ }^{8}$ Anti-inflammatory mechanism of lupeol also inhibited LPS-induced IкB $\alpha$ /degradation and the DNA binding activity of NFKB in IECs and macrophages. ${ }^{9}$

According to in vitro and silico screening of lupeol molecule have five targets side COX-2 (PDB ID: 4COX), MPO (PDB ID: 3ZS0), IL1 $\beta$ (PDBID" 1T4Q), IL6 (PDBID: 7 PM) and TNF $\alpha$ (PDBID: 2AZ5) which are capable for the anti-inflammatory activity and their auto dock results $(-11.6,-9.0,-9.9,-7.5,-9.0$ $\mathrm{kcal} / \mathrm{mol}$ ) showed that lupeol have maximum binding affinity. ${ }^{10}$

Dextran Sodium sulfate (DSS) leads to chronic inflammation and their protein binding site is TNF- $\alpha$, IL 1 and 2, and NF$\kappa B$. Lupeol has an anti-inflammatory agent which is used in controlling colitis and healing the colonic activity. ${ }^{11}$ Natural compound Lup-20(29)-en-3 $\beta$-ol has the potency to inhibit cytokine production, which is beneficial to inflammatory 
bowel diseases (IBD).12 Lupeol triterpenoids are used to treatment of bronchial asthma inflammatory diseases. It reduces the production of cellularity and eosinophils in the lungs of broncho-alveolar lavage fluid. ${ }^{13}$

\section{Lupeol act as anti-cancerous}

The present study of lupeol is reported as anticancer activity. Lupeol has been potential to act against different types of cancers such as human prostate, breast cancer skin, liver, and blood cancer. Different types of cancer have different cell lines like normal human breast cell line (MCF-10A) and cancer line MCF-7. A compound of lupeol induced in the cell line and changes the cell viability of MCF-7 with its IC50 concentration as $80 \mu \mathrm{M}$. The various striking observation of lupeol does not cause any toxic effect on a human cell it kills only cancerous cells. ${ }^{14}$ Vasculogenic mimicry and tumor microcirculation is found in many cancer stem-like cells. Recently anticancer of lupeol, a novel physiochemical with Dacarbazine both are in vivo and in vitro. Lupeol can become a more power full anticancer agent that treated the B16-F10 cell line and also inhibit the vasculogenic mimicry with inducing Dacarbazine drug resistance. ${ }^{15}$ Lung cancer is treated with the exploration and evaluation of new molecules of lupeol. Lupeol effects on lung cancer A427 cancer cells and normal MRC-5 cells. Observation of inhibiting the growth of lung cancer cells is checked by MTT assay. ${ }^{16}$ According to a worldwide report, cervical cancer is the most common cancer in women. Lupeol has been shown its anticancer activity in human cervical carcinoma(HeLa) cells and inhibitory activity through induction of S-phase cell cycle arrestand apoptosis. ${ }^{17}$ Colorectal cancer (CRC) is the main cause of death due to metastasis(CRC) patients. Effect of Lupeol treatment on colorectal cancer cell lines, HCT116and SW620 and suppress the migration of colorectal cancer cells by cytoskeleton RhoA-ROCK1 pathway inhibition, which provides an anti-metastatic activity forCRC patients ${ }^{18}$. In head and neck cancer (HNC) there is an overexpressed Epidermal growth factor receptor (EGFR) pathway. Lupeol-induced antitumor response is evaluated in two oral squamous cell carcinoma (OSCC) cell lines (UPCI:SCC131 and UPCI:SCC084). Lupeol inhibited the EGFR signaling in OSCC and it has a significant role in triggering antitumor efficacy. ${ }^{19}$ Lupeol effects on humannon-small cell lung cancer (NSCLC) and also suppressed the formation of NSCLC cells and inhibited the phosphorylation of epithelial growth factor receptor (EGFR). ${ }^{20}$

\section{Lupeol act as Anti-Diabetic}

Diabetes mellitus commonly known as diabetes, and a group of metabolic syndrome that causes high blood sugar level over a prolonged period. Diabetes is mainly of two types Type- 1 and Type-2.Lupeol has been reported to act as an anti-diabetic activity.21 Triterpenes of lupeol showed its activity to reduce the hyperinsulinemia through the regulation of insulin receptor and GLUT 4 protein. ${ }^{22}$ Lupeol study identifies the effects on enzymic antioxidants superoxide dismutase (SOD), catalase (CAT), and nonenzymic antioxidant (Vitamin C) in type-2 diabetic adult male rats and decrease the levels of antioxidant enzymes (SOD, CAT, and Vitamin C) in the liver of the type- 2 diabetic rats. Lupeol showed similar effects of metformin and regulates the antioxidant enzymes. ${ }^{23}$ Lupeol phytoconstituent from Solanum xanthocarpum, suppresses diabetes after 21 days. Lupeol also decreases glycated hemoglobin, serum glucose, and nitric oxide. ${ }^{24}$ In silico study is used to an interaction between molecules and ligand to predict three-dimensional structures. Many studies showed that glucose-lowering effects by plant extracts. Lupeol and iso-orientin are used as antidiabetic agents. The hypoglycemic activities of lupeol and iso-orientin areevaluated and confirmed in a rat model and extracts (either individually or in combination) significantly lowering blood glucose levels oxidative stress. ${ }^{25}$ Plants extract of lupeol inhibited the blood glucose levels in streptozotocin (STZ)-induced diabetic rats. ${ }^{26}$ Lupeol is alsoused for the treatment of diabetes which inhibited the alpha-glucosidase activity. Italso inhibited the alpha-amylase enzyme which is responsible for the development of diabetes. $^{27}$

\section{Lupeol act as cardioprotective}

Hyperlipidemia is a major risk factor to increase the level of cholesterol and the development of heart disease and various myocardial events. Plant sterols are beneficial and potential for lowering plasma cholesterol levels. Lupeol and its ester lupeol linoleate, are used to reduce the levels of hypercholesterolemic in the rats and decreases the activities of such enzymes namely $\mathrm{Na}+, \mathrm{K}+-\mathrm{ATPa} e, \mathrm{Ca} 2+-\mathrm{ATPase}$ and $\mathrm{Mg} 2+{ }^{28}$ Lupeol isolatedfrom Crataeva nurvala stem and preserve lysosomal integrity and improve the thiollevels, for their protective effect against CP-induced cardiotoxicity. ${ }^{29}$ Lupeol has pharmacological efficacy against CP-induced cardiac damage and restoration of mitochondrial structure and function. ${ }^{30}$ Lupeol treatment has a positive effect on high blood pressure and heart malfunction in male albino Wistar rats. It has also reestablished the levels of lipoproteins and improve the HDL cholesterol levels in dyslipidaemic. ${ }^{31}$ The complexes of triglycerides (TG), cholesterol, phospholipids, and apolipoproteins, also termed as lipoproteins are responsible for the transport of lipids in the bloodstream. There are fourmajor types of lipoproteins, (VLDL), (LDL), (HDL) and predicting the risk of atherosclerotic events. Lupeol decreases triglyceride and cholesterol secretion from HepG2-Lipo human hepatoma cells also suppresses the expressions of sterol regulatory element-binding protein-1c and -2, fatty acid synthase, 3-hydroxy-3- methylglutarylCoenzyme A synthetase-1, and farnesyl- diphosphate farnesyl transferase-1. Lupeol inhibited apolipoproteinB-100 present in the cells at the mRNA level. ${ }^{32}$ Lupeol and lupeol linoleate reduced the LPO levels and enhanced enzymatic and non-enzymatic antioxidants. These advantageous effects of lupeol and its linoleate ester derivatives are helpfulin hypercholesterolemia and atherosclerosis. ${ }^{33}$ Cardiac nuclear factor $\kappa \mathrm{B}$ and tumor necrosis factor- $\alpha$ levels increase the hypercholesterolemic condition and decreased nuclear factor- $\mathrm{kB}$ and oxidative stress with lupeol supplements. ${ }^{34}$ Lupeol has been shown to have cardioprotective effects and the study of lupeol was examined on the standard mouse model of CVB3 induced viral myocarditis. Lupeol may cause inhibition of the TLR4/MyD88/NF- $\mathrm{B}$ P65 signaling pathway and have the potential for the treatment of viral-induced myocarditis. ${ }^{35}$

\section{Lupeol act as skin protective}

Lupeol has been used to treat various skin ailments. We studied the beneficial effects of Lupeol in TPA-mediated cutaneous edema in the CD-1 mouse skin model. Lupeol before TPA application to mouse skin inhibited TPA-induced $\mathrm{NF}-\kappa \mathrm{B}, \quad \mathrm{IKK} \alpha$ activation, and phosphorylation and degradation of $\mathrm{I} \kappa \mathrm{B} \alpha$ protein Phosphorylation of $\mathrm{I} \kappa \mathrm{B} \alpha$. Lupeol inhibits $\mathrm{I} \kappa \mathrm{B} \alpha$ phosphorylation and degradation, of Lupeol on NF- $\kappa \mathrm{B} / \mathrm{p} 65$ is through the inhibition of proteolysis and $\mathrm{I} \kappa \mathrm{B} \alpha{ }^{36}$ Lupeol inhibits of tumor induction in murine skin and also suppresses free radical damage such as DNA, proteins. Benzoyl peroxide, a known free radical generating tumor enhancer was significantly prohibited by lupeol, when tested on murine skin in vivo. It acts as an effective chemopreventive agent against cutaneous toxicity. 37 One study evaluated the inhibitory effect of lupeol into two 
combination phytochemicals; pterostilbene on Swiss albino mice skin and combined treatment of phytochemicals was found to have better potential to prevent skin carcinogenesis 38 . Lupeol provides strong antioxidant protection against benzoyl peroxide-induced toxicity in Swiss albino mouse skin reduces the PGE2 production and inhibits the production of $\mathrm{TNF} \alpha$ and interleukin-1b in vitro. Lupeol showed significant anti-tumor promoting activity in a two-stage model of mouse skin carcinogenesis. Lupeol inhibits the activity of ornithine decarboxylase, and sensitive cells such as HeLa (cervix carcinoma) and A431 (skin carcinoma). ${ }^{39}$ Lupeol on 7,12 (DMBA)-induced in the skin of Swiss albino mice and showed significant ( $p<0.05)$ preventive effects against DMBA-mediated neoplastic Lupeol also inhibits the growth of cell-cycle regulation and apoptosis. ${ }^{40}$ DNA strand breaks genetic disorders including cancer and lupeol effect on 7,12- (DMBA), induced DNA strand breaks in mouse skin,by using an alkaline assay. The results suggest the preventive effects of lupeol on DMBA induced DNA alkylation damage in Swiss albino mice.41 Lupeol, its acetate, betulin, and betulinic acid inhibited the inflammatory activity induced by TPA in mice. Lupeol 3acetate and betulin decreased the tumor-promoting effect of TPA in mouse skin and more inhibitory compound quercetin, are an inhibitor of tumor promotion. lupeol 3-acetate and betulin inhibited the promotion of skin tumors of betulinic acid. ${ }^{22} \beta$-Lupeol has inhibited the growth of leukemia cells and also inhibited the mouse skin carcinogenesis in both prostate and pancreatic cancer. ${ }^{43}$ Lupeol exhibits significant inhibition of 20 prostate cancer cells proliferation and 21 also shows promising anti-tumor-promoting activity in a two 22 stage model of mouse skin carcinogenesis. ${ }^{44}$ Lupeol is a pharmaceutically satisfactory drug and used to improve the skin appearance and remove the fungal infections. ${ }^{45}$ Lupeol (Lup-20(29)-en-3b-ol), on CD-1 mouse skin, showed reduced 12-0-tetradecanoyl-phorbol-13-acetate- induced tumor incidence by the inhibition of phosphatidylinositol 3-kinase (PI3K), Akt, Nuclear factor kappaB (NFkB) and activation of Inhibitory- $\kappa B$ kinase- $\alpha$ (IKK- $\alpha) .{ }^{46}$

\section{Lupeol acts as an antiprotozoal agent}

Lupeol is also reported against many pathogenic protozoa. Many compounds are isolated from the medicinal plants for antimalarial activity against Plasmodium falciparum. ${ }^{47}$ The antiplasmodial activity of lupeol is by inhibition of schizont maturation with using 3D7 Plasmodium strains. It exhibits significant antiplasmodial effects with an $\mathrm{IC}_{50}$ value of $18 \mu \mathrm{g} / \mathrm{ml}$ and $3.8 \mu \mathrm{g} / \mathrm{ml}$, respectively. ${ }^{48}$ Lupeol showed antileishmanial activity against promastigote and amastigote forms. Lupeol damages the cytoplasmic membrane and suppress nitric oxide (NO) production and inhibit proinflammatory responses. It also induces NO production in L.donovani-infected macrophages and reduces the hepatic and splenic parasite. ${ }^{49}$ Lupeol has the potential to change mitochondrial swelling by sesquiterpene lactones against protozoan parasites and 4-nitrobenzaldehyde thiosemicarbazone inhibits the growth of promastigote and amastigote. 50 Lupeol inhibited erythrocyte prohibitive Plasmodium falciparum invasion and growth have inhibitory with $\mathrm{IC}_{50}$ values in the range 7-28 $\mathrm{mM}$. Lupeol offers specific antiplasmodial activity and have a novel target for antimalarial drugs. ${ }^{51}$ Lupeol exhibited antiparasitic activity of leaf extracts of Aerva lanata and Albendazole, also used for the treatment of parasite infections. ${ }^{52}$ Lupeol was investigated for anthelmintic activity against $T$. colubriformis, $H$. contortus and C. elegans from the Extracts of Curtisia dentate.It was active only for high concentrations of 1000 and $2000 \mu \mathrm{g} / \mathrm{ml}$, respectively. ${ }^{53}$

\section{Lupeol act as antimicrobial agents}

Lupeol has been found to act antimicrobial activity against microbes and enhancing the activity of antibiotics against methicillin-resistant Staphylococcus aureus (MRSA) also suppresses the minimum inhibitory concentration of several antibiotics against MRSA. ${ }^{54}$ Lupeol exhibits antimicrobial activity when tested for both Gram-positive and Gramnegative bacteria especially against C. Albicans. 55 Antimicrobial activity of lupeol containing Visnea mocanera leaves extract also exhibited anti-viral activity. ${ }^{56}$ Lupeol has been reported to high anti-viral activity against Herpes simplex virus- 1 (HSV1) or (HSV-2) infection. ${ }^{57}$ Lupeol is compared for its anti-viral activity with acyclovir on the growth of the type strain of HSV-1. Monolayers of Vero E6 cells were infected with HSV-1 and treated with lupeol concentrations $(0-10 \mu \mathrm{g} / \mathrm{ml})$ and also inhibited the growth of plaque formation. ${ }^{58}$ Lupeol showed that it inhibits the HIV-1 reverse transcriptase (RT)-associated RNA-dependent DNA polymerase (RDDP) activity and antiviral properties of compounds against the $\alpha$ - glucosidase. ${ }^{59}$ Lupeol extracted from Maytenus genus exhibited potent antiviral activity against Dengue virus and low cytotoxicity in LLC-MK2cells. ${ }^{60}$

\section{Lupeol act as a nephroprotective agent}

The role of lupeol has been tested for its protective efficacy against renal toxicity and anti-urolithiasis activity. Lupeol decreases the levels of calcium-oxalate and has cytoprotective action against free- radical-induced damage and also decreases the level of Cadmium in the kidney. 61 Renal cell carcinoma (RCC) is kidney cancer in epithelial proximal tubular cells. Lupeol has effects on SKRC-45 (an RCC cell line) and has potential against RCC within mitochondrial dynamics. ${ }^{62}$ Lupeol reduced the crystal deposition in the kidneys such as calcium, oxalate and uric acid and also decreased the concentration of inhibitors, such as magnesium and glycosaminoglycans, deposition. 63 Animals were treated with $2 \%$ solution of ammonium oxalate for 15 days inducing hyperoxaluric condition in rats. Lupeol restored the level of renal enzymes and increased urinary excreation. ${ }^{64}$ In vitro antiurolithiatic activity of lupeol was evaluated to inhibit calcium oxalate and nucleation aggregation at different concentrations of extract/fractions (0.04-3 mg/mL) for 30 min. ${ }^{65}$

\section{LUPEOL DERIVATIVES BY SEMISYNTHESIS}

Lupeol extracted from the leaves of Aegle marmelos and their ester derivatives at $\mathrm{C}-3$ position are used to antihyperglycemic activity. 66 


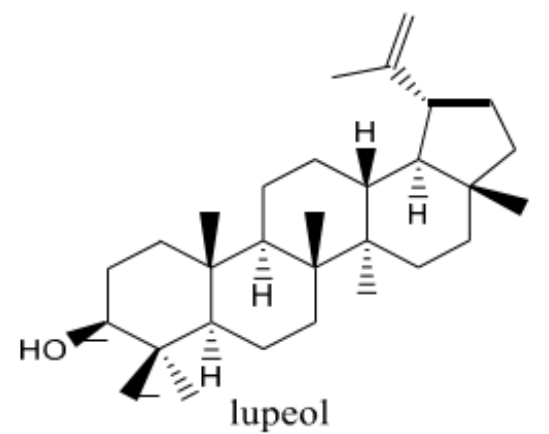

Appropriate acid<smiles>[Mg][Mg][Mg][Mg]Cl</smiles>

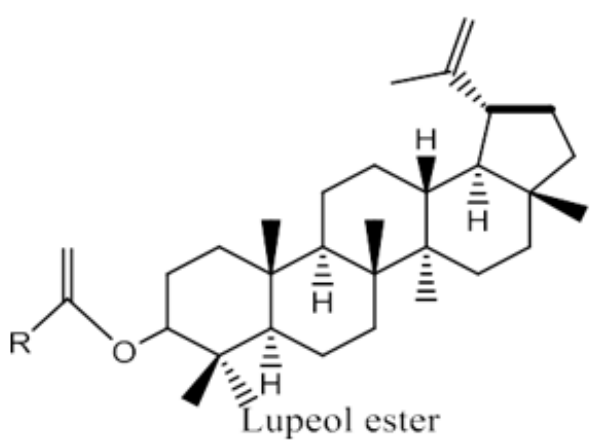

Lupeol isolated from the stem bark of Bombax ceiba and some derivatives of lupeol at C-3 and C-29position are synthesized for antitumor activities.67
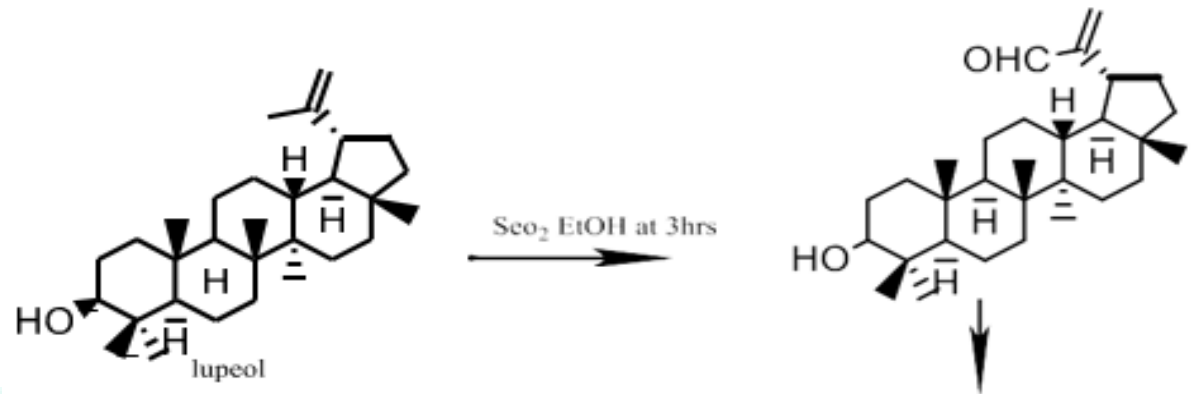<smiles>OCC=CC1CC1</smiles>
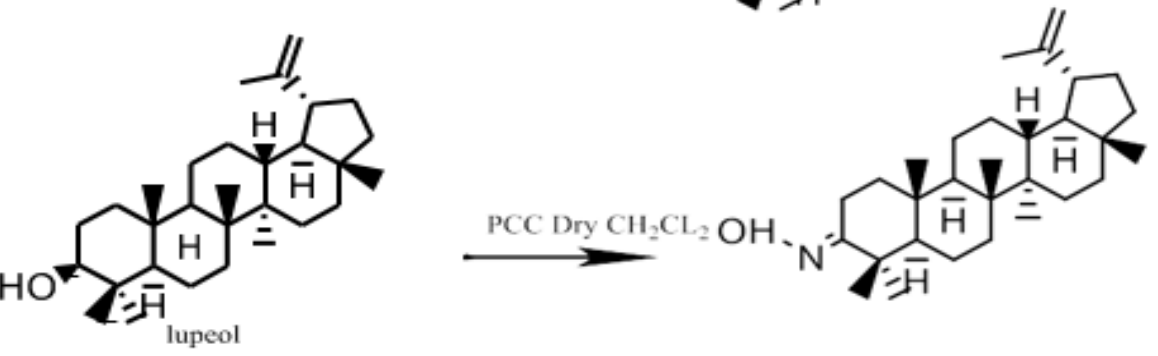

Synthesis of lupeol derivatives

Lupeol was isolated from aerial parts of $V$. scorpioides and modification at C-3 and C-29 position their derivatives are used for lymphoid leukemia cell activity. 68
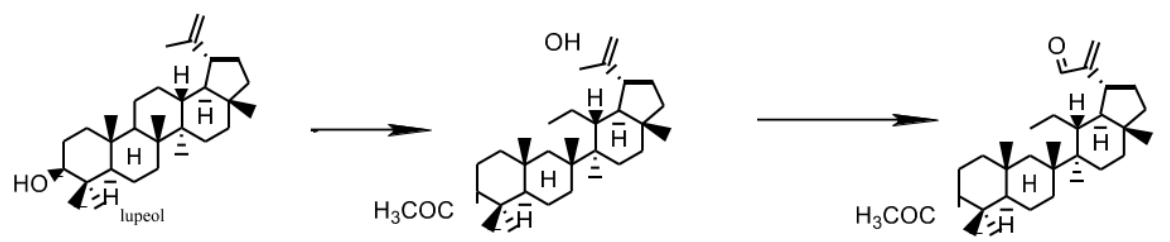

Lupeol extract of Walsura trifoliate and novel derivatives are synthesized for anti-proliferative activity against MDAMB231, IMR32and A549 cell lines. 69 

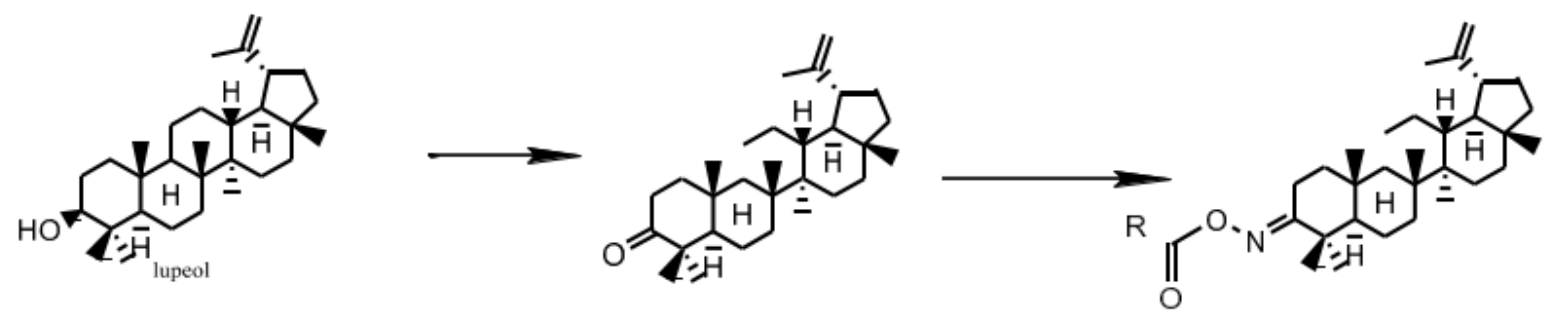

Oxime ester derivatives

Lupeol ester derivatives are synthesized for the treatment of skin damages activity. ${ }^{70}$

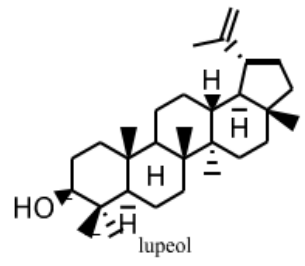

\section{CONCLUSION}

A pentacyclic triterpenoid i.e. Lupeol is widely distributed in the plant kingdom and is found in various fruits and vegetables. This naturally occurring triterpene is found effective in case of inflammatory responses and have immunomodulatory properties. As this review demonstrates, lupeol and some analogs have been shown to possess a series of folk and recognized biological activities, and further the potential to be consumed as a nutritive supplement to prevent cancer, inflammation, coronary and hepatic diseases. Lupeol also exhibited low cytotoxicity on healthy cells and acted synergistically when used in combined therapies, which make it worthy of exploration to be employed alone or as an adjuvant to clinically used antineoplastic and antiinflammatory drugs.

\section{ACKNOWLEDGEMENT}

The authors are grateful to the Abhilashi University, Mandi, $\mathrm{HP}$, India for providing various facilities in connection with this work.

\section{CONFLICT OF INTEREST}

The authors declare no conflict of interest, financial or otherwise.

\section{REFERENCES:}

1. Rao SD, Rao BN, Devi PU, Rao AK. Isolation of Lupeol, Design and Synthesi of Lupeol Derivatives and their Biological Activity. Oriental Journal of Chemistry. 2017; 33(1):173-80.

2. Patocka J.Biologically active pentacyclic triterpenes and their current medicine signification. Journal of Applied Biomedicine. 2003; 1(1):7-12.

3. Variya, Bhavesh C, Bakrania Anita, K Patel, Snehal S. Emblica officinalis (Amla): A review for its phytochemistry, ethnomedicinal uses and medicinal potentials with respect to molecular mechanisms. Pharmacological research. 2016; 111:180-200.

4. Phyllanthus, emblica, Germplasm, Resources information Network (GRIN). Agricultural Research Service (ARS), United States Departments of Agriculture (USDA). Retrieved 200803-06.

5. Siddique, Hifzur Rahman, Saleem Mohammad. Beneficial health effects of lupeol triterpene: a review of preclinical studies. Life sciences. 2011; 88(7-8):285-293.

6. Saleem, Mohammad. Lupeol, a novel anti-inflammatory and
$\mathrm{H}$

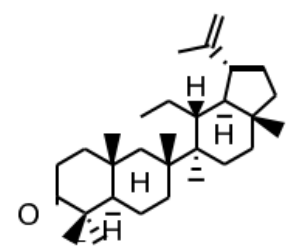

Ester derivatives anti- cancer dietary triterpene. Cancer letters. 2009; 285(2):109-115.

7. Fernández, Maria Angeles, et al.New insights into the mechanism of action of the anti.inflammatory triterpene lupeol. Journal of Pharmacy and Pharmacology. 2001; 53(11):1533-1539.

8. Oliveira-Junior, Markley Silva, et al. Lupeol inhibits LPSinduced neuroinflammation in cerebellar cultures andinducesneuroprotection associated to the modulation of astrocyte response and expression of neurotrophic and inflammatory factors. International immunopharmacology. 2019; 70:302-312.

9. Lee, Changhyun, et al. Lupeol inhibits LPS-induced NF-kappa B signaling in intestinal epithelial cells and macrophages, and attenuates acute and chronic murine colitis. Life sciences. 2016; 146:100-108.

10. Thirumalaisamy, R., et al.In-Vitro and In-Silico Antiinflammatory Activity of Lupeol Isolated from Crateva adansonii and Its Hidden Molecular Mechanism. International Journal of Peptide Research and Therapeutics. 2020:1-11.

11. Kasinathan, Nirmal Kumar; Subramaniya, Bharathraja; Sivasithamparam, Niranjali Devaraj. NF-kB/twist mediated regulation of colonic inflammation by lupeol in abating dextran sodium sulfate induced colitis in mice. Journal of functional foods. 2018; 41:240-249.

12. Zhu, Yeshan, et al. The pentacyclic triterpene Lupeol switches M1 macrophages to M2 and ameliorates experimental inflammatory bowel disease. International immunopharmacology. 2016; 30:74-84.

13. Vasconcelos, Juliana Fraga, et al.The triterpenoid lupeol attenuates allergic airway inflammation in a murine model. International immunopharmacology. 2008; (8):12161221.

14. Pitchai Daisy, Roy Anita, Ignatius Cybil. In vitro evaluation of anticancer potentials of lupeol isolated from Elephantopus scaber L. on MCF-7 cell line. Journal of advanced pharmaceutical technology \& research. 2014; (5):179.

15. Bhattacharyya Sayantan, et al. Reversing effect of Lupeol on vasculogenic mimicry in murinemelanomaprogression. Microvascular research. 2019; 121:52-62.

16. HE Wei, LI Xiang, XIA Shuyue. Lupeol triterpene exhibits potent antitumor effects in A427 human lung carcinoma cells via mitochondrial mediated apoptosis, ROS generation, loss of mitochondrial membrane potential and downregulation of $\mathrm{m}$ TOR/PI3Ksol; AKT signalling pathway. J BUON. 2018; 23:635640.

17. Prasad Nupoor, et al. Lupeol induces S-phase arrest and mitochondria-mediated apoptosis in cervical cancer cells. Journal of biosciences, 2018; 43(2):249-261.

18. Jiang Yiwen, et al. Lupeol inhibits migration and invasion of 
colorectal cancer cells by suppressing RhoA-ROCK1 signaling pathway. Naunyn-Schmiedeberg's Archives of Pharmacology. 2020:1-12.

19. Rauth Sanchita, et al. Lupeol evokes anticancer effects in oral squamous cell carcinoma by inhibiting oncogenic EGFR pathway. Molecular and cellular biochemistry. 2016; 417(12):97-110.

20. MinTae-Rin, et al. Suppression of EGFR/STAT3 activity by lupeol contributes to the induction of the apoptosis of human non-small cell lung cancer cells. International Journal of Oncology. 2019; 55(1):320-330.

21. Soni Lokesh Kumar, et al. In vitro and in vivo antidiabetic activity of isolated fraction of Prosopis cineraria against streptozotocin-induced experimental diabetes: A mechanistic study. Biomedicine \& Pharmacotherapy. 2018; 108:10151021.

22. Shreenithi S., et al. In silico and in vivo approach to identify the antidiabetic activity of lupeol. Drug Invention Today. 2019; 11:5.

23. Pushpanjali G., et al. Effect of lupeol on enzymatic and nonenzymatic antioxidants in type-2 diabetic adult male Wistar rats. Drug Invention Today. 2019; 12:5.

24. Gupta Rajnish, et al. Evaluation of antidiabetic and antioxidant potential of lupeol in experimental hyperglycaemia. Natural product research. 2012; 26(12):1125-1129.

25. Malik Arif, et al. In silico and in vitro studies of lupeol and isoorientin as potential antidiabetic agents in a rat model. Drug design, development and therapy. 2019; 13:1501.

26. Narváez-Mastache, José M., et al. Antihyperglycemic activity and chemical constituents of Eysenhardtia platycarpa. Journal of natural products. 2006; 69(12):1687-1691.

27. Ortiz-Andrade, RR., et al. $\alpha$-Glucosidase inhibitory activity of the methanolic extract from Tournefortia hartwegiana: an anti- hyperglycemic agent. Journal of ethnopharmacology. 2007; 109(1):48-53.

28. Sudhahar, Varatharajan, et al. Protective effect of lupeol and its ester on cardiac abnormalities in experimental hypercholesterolemia. Vascular pharmacology. 2007; 46(6):412-418.

29. Sudharsan, Periyasamy Thandavan, et al. Lupeol and its ester ameliorate the cyclophosphamide provoked cardiac lysosomal damage studied in rat. Molecular and cellular biochemistry. 2006; 282(23):1-2.

30. Sudharsan, Periyasamy Thandavan, et al. Lupeol and its ester exhibit protective role against cyclophosphamide-induced cardiac mitochondrial toxicity. Journal of cardiovascular pharmacology.2006; 47(2):205-210.

31. Thilakarathna Surangi H,Rupasinghe, HP Vasantha. Antiatherosclerotic effects of fruit bioactive compounds: A review of current scientific evidence. Canadian Journal of Plant Science. 2012; 92(3):407-419.

32. Itoh, Mizuho, et al. Lupeol reduces triglyceride and cholesterol synthesis in human hepatoma cells. Phytochemistry Letters. 2009; 2(4):176-178.

33. Sudhahar, Varatharajan,Kumar Sekar Ashok,Varalakshmi Palaninathan. Role of lupeol and lupeol linoleate on lipemicoxidative stress in experimental hypercholesterolemia. Life sciences. 2006; 78(12):1329-1335.

34. SudhaharVaratharajan, et al. Remedial effect of lupeol and its ester derivative on hypercholesterolemia-induced oxidative and inflammatory stresses. Nutrition Research. 2007; 27(12):778-787.

35. XU Ming, et al. Lupeol alleviates coxsackievirus B3-induced viral myocarditis in mice via downregulating toll-like receptor 4. Journal of International Medical Research. 2020; 48(4):0300060520910908.

36. Saleem Mohammad, et al.Lupeol modulates NF- $\kappa$ B and PI3K/Akt pathways and inhibits skin cancer in CD-1 mice. Oncogene. 2004; 23(30):5203-5214.

37. Sultana Sarwat, et al. Lupeol, a triterpene, prevents free radical mediated macromolecular damage and alleviates benzoyl peroxide induced biochemical alterations in murine skin.Indian journal of experimental biology.2003; 41(8):82731.

38. Singh Payal, Arora Deepika,Shukla Yogeshwer. Enhanced chemoprevention by the combined treatment of pterostilbene and lupeol inB[a]P-induced mouse skin tumorigenesis. Food and Chemical Toxicology. 2017; 99:182-189.
39. Ciurlea Sorina, et al.Lupeol, a pentacylcic triterpene that reduces the lesions and irritability on murine skin and is effective on in vitro tumormodels.Journal of Agroalimentary Processes and Technologies. 2010; 16(4):427-432.

40. NigamNidhi, et al. Lupeol induces p53 and cyclin-B-mediated $\mathrm{G} 2 / \mathrm{M}$ arrest and targets apoptosis through activation of caspase in mouse skin. Biochemical and biophysical research communications. 2009; 381(2):253-258.

41. Nigam Nidhi,Prasad Sahdeo,ShuklaYogeshwer. Preventive effects of lupeol on DMBA induced DNA alkylationdamage in mouse skin. Food and chemical toxicology. 2007; 45(11):2331-2335.

42. Yasukawa K., et al. Some lupane-type triterpenes inhibit tumor promotion by 12-0-tetradecanoylphorbol-13-acetate in twostage carcinogenesis in mouse skin. Phytomedicine. 1995; 1(4):309-313.

43. Chávez-Piña Aracely et al. Gastroprotective effect of $\beta$ - LupeoL: roLe of prostaGLandins, suLfhydryLs and nitric oxide. Revista Latinoamericana de Química. 2009; 37(2):133-143.

44. SrivastavaAmit Kumar, et al. Protective effects of lupeol against mancozeb-induced genotoxicity in cultured human lymphocytes. Phytomedicine. 2016; 23(7):714-724.

45. Gibson David J, Carlson Robert M. Methods of treating fungal infections using lupeol. United States Reexamination Certificate Second Reexamination US20030431351. 07 May 2003.

46. Prasad Sahdeo, et al. Induction of apoptosis by lupeol in human epidermoid carcinoma A431 cells through regulation of mitochondrial, Akt/PKB and NF-kappaB signaling pathways. Cancer biology \& therapy. 2009; 8(17):1632-1639.

47. Khalid Sami A., et al. Potential antimalarial candidates from African plants: an in vitro approach using Plasmodium falciparum. Journal of Ethnopharmacology. 1986; 15(2):201209.

48. Singh Amandeep, et al. Investigation of antiplasmodial efficacy of lupeol and ursolic acid isolated from Ficus benjamina leaves extract. Natural product research. 2018:1-4.

49. Das Antu, et al. Antileishmanial and immunomodulatory activities of lupeol, a triterpene compound isolated from Sterculia villosa. International journal of antimicrobial agents. 2017; 50(4):512-522.

50. IsahMurtala Bindawa, et al. Terpenoids as Emerging Therapeutic Agents: Cellular Targets and Mechanisms of Action against Protozoan Parasites. Studies in Natural Products Chemistry. 2018:227-250.

51. Ziegler Hanne L., et al. Erythrocyte membrane modifying agents and the inhibition of Plasmodium falciparum growth: structure-activity relationships for betulinic acid analogues. Bioorganic \& medicinal chemistry. 2004; 12(1):119-127.

52. Goyal Manoj, et al. Aerva lanata: A review on phytochemistry and pharmacological aspects. Pharmacognosy reviews. 2011; 5(10):195.

53. Shai Leshweni Jeremia et al. Curtisia dentata (Cornaceae) leaf extracts and isolated compounds inhibit motility of parasitic and free- living nematodes. Onderstepoort Journal of Veterinary Research. 2009; 76(2):249-256.

54. Okusa Philippe N., et al. Ferulaldehyde and lupeol as direct and indirect antimicrobial compounds from Cordia gilletii (Boraginaceae) root barks. Natural product communications, 2014; 9(5):1934578X1400900506.

55. Amoussa Abdou Madjid 0., et al. Triterpenoids from Acacia ataxacantha DC: antimicrobial and antioxidant activities. Complementary and alternative medicine. 2016; 16(1):284.

56. Hernández-Pérez M., et al. Antimicrobial activity of Visnea mocanera leaf extracts. Journal of ethnopharmacology. 1994; 41(1-2):115-119.

57. Mutai C., et al. Effects of Triterpenoids on Herpes Simplex Virus Type1 (Hsv-1).2012.

58. Tolo FM., et al. The antiviral activity of compounds isolated from Kenyan Carissa edulis (Forssk.) Vahl. JMPR.2010; $4(15): 1517-22$.

59. EspositoFrancesca, et al. Multi-target activity of Hemidesmus indicus decoction against innovative HIV-1 drug targets and characterization of Lupeol mode of action. Pathogens and disease. 2017; 75(6):65.

60. Silva Fernando C., et al. Antidiarrheal activity of extracts from Maytenus gonoclada and inhibition of Dengue virus by lupeol. Anais da Academia Brasileira de Ciências. 2017; 89(3):1555- 
1564.

61. Nagaraj M, Sunitha S,VaralakshmiP. Effect of lupeol, a pentacyclic triterpene, on the lipid peroxidation and antioxidant status in rat kidney after chronic cadmium exposure. Journal of Applied Toxicology: An International Journal. 2000; 20(5):413-417.

62. SinhaKrishnendu, et al. Lupeol alters viability of SK-RC-45 (Renal cell carcinoma cell line) by modulating its mitochondrial dynamics. Heliyon. 2019; 5(8):e02107.

63. Vidya L, Varalakshmi P. Control of urinary risk factors of stones by betulin and lupeol in experimental hyperoxaluria. Fitoterapia. 2000; 71(5):535-543.

64. Malini Mariappan M, Baskar Ramakrishnan, Varalakshmi Palaninathan. Effect of lupeol, a pentacyclic triterpene, on urinary enzymes in hyperoxaluric rats. Japanese Journal of Medical Science and Biology. 1995; 48(5):211-220.

65. DevkarRaviraj Anand, et al. Evaluation of antiurolithiatic and antioxidant potential of Lepidagathis prostrata: aPashanbhed plant. Pharmaceutical biology. 2016; 54(7):1237-1245.

66. Reddy KPapi, et al. Synthesis of novel triterpenoid (lupeol) derivatives and their in vivo antihyperglycemic and antidyslipidemic activity. Bioorganic \& medicinal chemistry letters.2009; 19(15):4463-4466.

67. Saini Monika, et al. Design, Synthesis and In-Vitro Antitumor Activity of LupeolDerivativesvia Modification atC.3 and C.30 Positions. ChemistrySelect. 2019; 4(5):1800-1805.

68. Machado, Vanessa Rocha, et al.Anti-leukemic activity of semisynthetic derivatives of lupeol. Natural Product Research. 2020:1-8.

69. S Devendra, et al. Isolation of lupeol, design and synthesis of lupeol derivatives and their biological activity. Oriental Journal of Chemistry. 2017; 33(1):173-180.

70. Malinowska M, Miroslaw B, Sikora E, et al. New lupeol esters as active substances in the treatment of skin damage. PLoS One. 2019; 14(3):e0214216. 